\title{
Musical mirror-symmetrical movement tasks: comparison of rhythm versus melody-playing Serhat Tokgoz ${ }^{a}$, Demet Aydogdu ${ }^{b}$, Barkin Ilhan ${ }^{c}$, Yusuf Sahin ${ }^{d}$, Nurtug Bariseri ${ }^{\mathrm{e}}$, Batu Mehmet Ozturkler ${ }^{\mathrm{f}, \mathrm{g}}$ and Tolga Çukur ${ }^{\mathrm{h}}$
}

\begin{abstract}
Bimanual mirror-symmetrical movement (MSM) is relatively easy to control movement. Different MSM tasks may have different activations and interhemispheric interactions. The purpose of this study is to compare anatomo-physiological features such as hemispheric activations and dominance of two different MSMs, namely melody-playing and rhythm. We examined functional MRI (fMRI) recordings in a group of fifteen right-handed pianists performing two separate tasks: bimanual rhythm and bimanual melody-playing on two different keyboards with standard key order for right hand and reversed for left hand, which allows homolog fingers' movements. Activations and laterality indices on fMRI were examined. The results show that significant cerebellar activations (especially in anterior cerebellum) in both groups. Significant primary sensorimotor cortical activations are observed in the melody-playing group. While there are also bilaterally symmetric activations, and laterality indices suggest overall lateralization towards the left hemisphere in both groups. Activations in the left fronto-parietal cortex, left putamen and left thalamus in conjunction with right cerebellar activations suggest that the left corticothalamo-cerebellar loop may be a dominant loop. Dynamic causal modeling (DCM) indicates the presence of causal
\end{abstract}

\section{Introduction}

In-phase movement (mirror-symmetrical movement, MSM) is a bimanual movement that is relatively easy to control tasks in many organisms. In MSMs, relaxed contralateral hand muscles are simply facilitated during voluntary contraction of the ipsilateral homolog muscle [1]. As such, in-phase movement can lead to decreased intercortical inhibition and increased excitatory effects [2]. Several in-phase tasks including bimanual voluntary tapping, mirror writing, mirror drawing, or navigation were examined in the literature [3-7]. These studies examined the effects of bimanual coordination on the stability of motor tasks and their neural mechanisms (Text 1, Supplemental Digital Content 1, http://links.lww.com/ WNR/A582). Taken together, prior literature indicates that bimanual in-phase MSMs elicit reduced responses compared to anti-phase and unimanual movements in a

Supplemental Digital Content is available for this article. Direct URL citations appear in the printed text and are provided in the HTML and PDF versions of this article on the journal's website, www.neuroreport.com. influences from the left to the right cerebral cortex. In conclusion, melody-playing with bimanual MSM is a complex in-phase task and may help activate the bilateral cortical areas, and left hemisphere is dominant according to laterality indices and DCM results. On the other hand, bimanual rhythm is a simpler in-phase task and may help activate subcortical areas, which might be independent of the voluntary cortical task. NeuroReport 31: 523-529 Copyright (c) 2020 Wolters Kluwer Health, Inc. All rights reserved.

\section{NeuroReport 2020, 31:523-529}

Keywords: cerebellum; motor control, in-phase movement, mirror-symmetrical movement

Departments of a Neurology, ${ }^{b}$ Radiology, ${ }^{c}$ Biophysics, Meram Medical Faculty, NecmettinErbakan University, Konya, Turkey, ${ }^{d}$ Department of Mechanical Engineering, Nigde University, Konya, Turkey, ' ${ }^{2}$ epartment of Music, Education Faculty, NecmettinErbakan University, Konya, Turkey, 'Department of Electrical Engineering, Stanford University, California, USA, ${ }^{9}$ Department of Electrical and Electronics Engineering, Middle East Technical University and ${ }^{\mathrm{h}}$ Department of Electrical and Electronics Engineering, Neuroscience Graduate Program, Bilkent University, National Magnetic Resonance Research Center, Ankara, Turkey

Correspondence to Serhat Tokgoz, MD, Department of Neurology, NecmettinErbakan University, Meram Medical Faculty, Konya, 42081 Turkey Tel: +90 332 2236785; fax: +90 332 2236181;

e-mail: osmanserhattokgoz@gmail.com

Received 30 January 2020 Accepted 15 February 2020

set of regions including left primary motor cortex (M1), right dorsal premotor cortex and supplementary motor area (SMA) and that interhemispheric talk at the level of M1 plays a role in movement stabilization.

Although bimanual MSMs are simple, stable motor movements, musical in-phase tasks remain understudied to the best of our knowledge. Therefore, the main motivation of the current study was to comparatively examine anatomo-physiological features -including interhemispheric crosstalk - of two musical bimanual MSMs in pianists, namely melody-playing and rhythm. Prior studies suggest a more efficient neural organization for hand motor control in pianists due to long-term motor practice [8] (see for a discussion Text 2, Supplemental Digital Content 1, http://links.lwrw.com/WNR/A582) [9-11]. Therefore, we recruited pianists here to increase sensitivity to potential differences in anatomo-physiological features between the MSM tasks.

Here, we consider a bimanual rhythm task that involves simple homolog finger movements. We also introduce 
bimanual piano playing, where homolog fingers play the same notes that significantly differs from prior methods [12-14] (see Text 2, Supplemental Digital Content 1, http://links.lww.com/WNR/A582) [15,16]. This in-phase melody-playing task involves bimanual movement where a regular keyboard for the right hand and a reversed keyboard for the left-hand is used (Supplemental Digital Content 2, http://links.lwwe.com/WNR/A583). Bimanual movement is transformed from anti-phase to in-phase movement similar to a 'phase transition' [17]. This allows the homolog fingers to play the same notes.

We performed fMRI on a group of fifteen right-handed pianists, where the group implemented both the bimanual rhythm task and the melody-playing task. Activations and laterality indices on fMRI were examined. Dynamic causal modeling (DCM) was performed where three different hypotheses were tested: modulation from only left-to-right, only right-to-left or in both directions. Based on experimental results, we discuss how the different in-phase tasks may contribute to bilateral activations in the scope of neurophysiology literature.

\section{Methods \\ Subjects}

This study included 15 (8 female/7 male) volunteering right-handed pianists with an average of 7 years (interquartile range: 5-7) of music experience (see for details Text 3, Supplemental Digital Content 1, http://links.lww. $\mathrm{com} / W N R / A 582)$ [18,19]. Approval forms and written informed consent were obtained from all participants. Experimental procedures were approved by the local ethics committee. The study was performed in accordance with the Declaration of Helsinki.

\section{Experimental design}

The subjects were asked to tap the rhythm and perform melody-playing with two keyboards. In our design, the notes on the keyboard used for left hand were symmetrical to the keyboard used for right hand, which allows subjects to play the same notes with the same fingers in the melody-playing as an in-phase MSM (Fig. 1). Having had no prior experience with the setup, the subjects could nevertheless play two keyboards with ease. Later, the subjects were immediately directed to fMRI examinations.

\section{Behavioral procedures}

The active phase consisted of two procedures:

(1) A simple known melody at a tempo of 55 beats per minute was used. Melody-playing with the flexion of the proximal interphalangeal joint of all fingers for sequential finger movements with imaginary keyboards was performed. Hands were placed near their knees.

(2) Rhythm tapping with the flexion of the proximal interphalangeal joint of all fingers simultaneously for all fingers (Text 3, Supplemental Digital Content 1, http://links.lww.com/WNR/A582 for further details).

\section{Functional MRI procedures}

Brain images were acquired on a 1.5 Tesla MRI device equipped with an eight-channel head coil (Magnetom Symphony, Siemens, Erlangen, Germany) in the supine position where the head was restrained via a standard head bandage. Whole-brain functional MRI data were collected with blood oxygen level-dependent (BOLD) contrast. Functional images were collected with a T2*weighted single-shot gradient echo-planar imaging sequence. The acquisition parameters for functional imaging were a flip angle of $90^{\circ}$, a time repetetion/time echo (TR/TE) of $4010 / 50 \mathrm{~ms}$, a number of excitations of 1, a field-of-view (FOV) of $211 \mathrm{~mm}$, a resolution of $3.3 \times 3.3 \times 3 \mathrm{~mm}^{3}$, and an interslice space of $0.75 \mathrm{~mm}$. A readout bandwidth of $1475 \mathrm{~Hz} /$ pixel was used. For each subject, fMRI data were acquired in a single series with 60 -time points, with a total scan time of $4 \mathrm{~min} 5 \mathrm{~s}$. Each series were composed of 3 active phase blocks with 10 images in each block, and 3 resting phase blocks with 10

Fig. 1

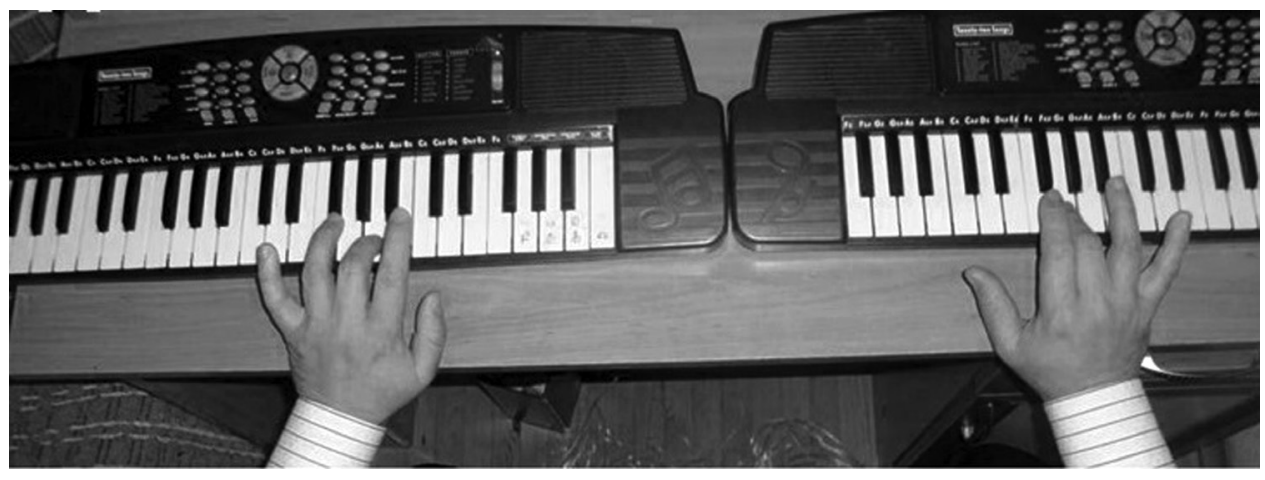

Recording procedure: Two keyboards (the left one modified reversely). 
images in each block. Anatomical images were collected with T1-weighted spin-echo pulse sequences. The acquisition parameters for anatomical imaging were a flip angle of $70^{\circ}$, a TR/TE of $500 / 12 \mathrm{~ms}$, a FOV of $211 \mathrm{~mm}$, a resolution of $1.1 \times 1.47 \times 3 \mathrm{~mm}^{3}$, and an interslice space of $0.75 \mathrm{~mm}$. Thirty-six slices were acquired. A readout bandwidth of $130 \mathrm{~Hz} /$ pixel was used. Each acquisition lasted $2 \mathrm{~min} 28 \mathrm{~s}$. Functional images were registered with T1-weighted anatomical images and functional mapping of brain anatomy was performed. See Text 3, Supplemental Digital Content 1, http://links.lww.com/ WNR/A582 for data preprocessing procedures.

\section{Statistical analysis for functional MRI General linear models}

Voxel-by-voxel statistical analysis was conducted on each subject using the general linear model to correlate the time course of measured BOLD signals with the reference function generated by a box-car function convolved with the hemodynamic response function. For all 15 subjects, 60 functional volumes were used. Task-related activations were evaluated using four different general linear models. The first model evaluated activations related to melody-playing via a contrast vector [ $\left[\begin{array}{ll}0 & 1\end{array}\right]$ between melody-playing and resting. The second model evaluated activations related to rhythm via a contrast vector of [0 1] between rhythm and resting. The third model used a contrast vector of $[1-1]$ to distinguish the activated brain regions between melody-playing and rhythm. The fourth model used a contrast vector of $\left[\begin{array}{ll}1 & -1\end{array}\right]$ to distinguish the activated brain regions between rhythm and melody-playing. For all general linear models, design matrices were constructed using the above-mentioned contrast vectors and the scan timing parameters. In all four models, cluster-forming thresholding was applied $(P<0.001)$. Each model was separately fit across the whole brain. Both individual-subject and group-level analyses were performed. For group-level assessment, a fixed-effects analysis was performed for improved sensitivity. Voxel time courses and design matrices were aggregated by concatenating the respective time courses across subjects. Voxelby-voxel statistical analysis was then conducted using the multi-subject time courses and the multi-subject design matrix as in the individual-subject case.

\section{Laterality index}

Laterality indices numerical data were imported into SPSS 15 (version 15.0: SPSS Inc, Chicago, Illinois, USA) for descriptive analysis and graphical visualization. Significance of differences between the melody-playing and rhythm tasks in predefined regions of interests were assessed with Mann-Whitney $U$ tests (see Text 3, Supplemental Digital Content 1, http://links.lwre.com/WNR/A582) [20].

\section{Dynamic causal modeling}

DCM was applied to assess connectivity between left and right hemispheres in melody-playing and rhythm (see Text 3, Supplemental Digital Content 1, http://links. lww.com/WNR/A582) [21,22]. Knowing both left and right hemispheres are activated, both tasks were specified as a direct effect on both hemispheres. Three different models, each of which corresponds to three different hypotheses, were tested:

(1) Task acts as modulatory input from left to right.

(2) Task acts as modulatory input from right to left.

(3) Task acts as modulatory input from both left to right and right to left.

For all three models, analyses were conducted by extracting and fitting time-series from left hemisphere and right hemisphere. Modulatory inputs were assumed to be bilinear. Number of states per each region was assumed to be one. Stochastic effects and center inputs were not considered. DCM was applied independently to all 15 subjects. Results were then averaged across subjects using Bayesian parameter averaging. Finally, to test the validity of these models Bayesian model comparison was applied. A threshold probability of 0.9 was used to determine whether direct or modulatory effects were present. To control for multiple comparisons, cluster-forming thresholding was applied $(P<0.001)$ at the voxel level.

\section{Results}

\section{Functional MRI findings}

Activations were evaluated for melody-playing vs. rest and rhythm vs. rest contrasts for individual subjects and for the group-level separately $(P<0.001)$. In the individual-subject analysis, for both melody-playing and rhythm tasks, bilateral activations in the primary sensorimotor cortices, the SMA, the anterior lobe of the cerebellum, left thalamus and left putamen were observed for all 15 subjects. For the rhythm task, activations in anterior lobe of the cerebellum, across superior vermis and paravermian areas were observed in 12 subjects. Moreover, distinctively for the melody-playing task as opposed to the rhythm task, PPC activation was observed for 9 subjects.

In the group-level analysis, in both melody-playing and rhythm tasks, significant activations were observed across cortical areas and the cerebellum. In both tasks, there were bilateral activations in the primary sensorimotor cortices, the SMA, the anterior lobe of the cerebellum, left thalamus and left putamen. In the melody-playing task, symmetric activations were present in the posterior parietal cortices (PPC) (Fig. 2a). Global maximal activation was in the right fronto-parietal cortices (Table 1). In the rhythm task, significant activations were observed in the anterior lobe of the cerebellum, across superior vermis and paravermian areas, partially spreading to the cortices (Fig. 2b). However, PPC activation, which was observed during the melody-playing task, was not observed. For the rhythm task, global maximal activation was in the anterior lobe of the cerebellum (Table 1). 
Fig. 2
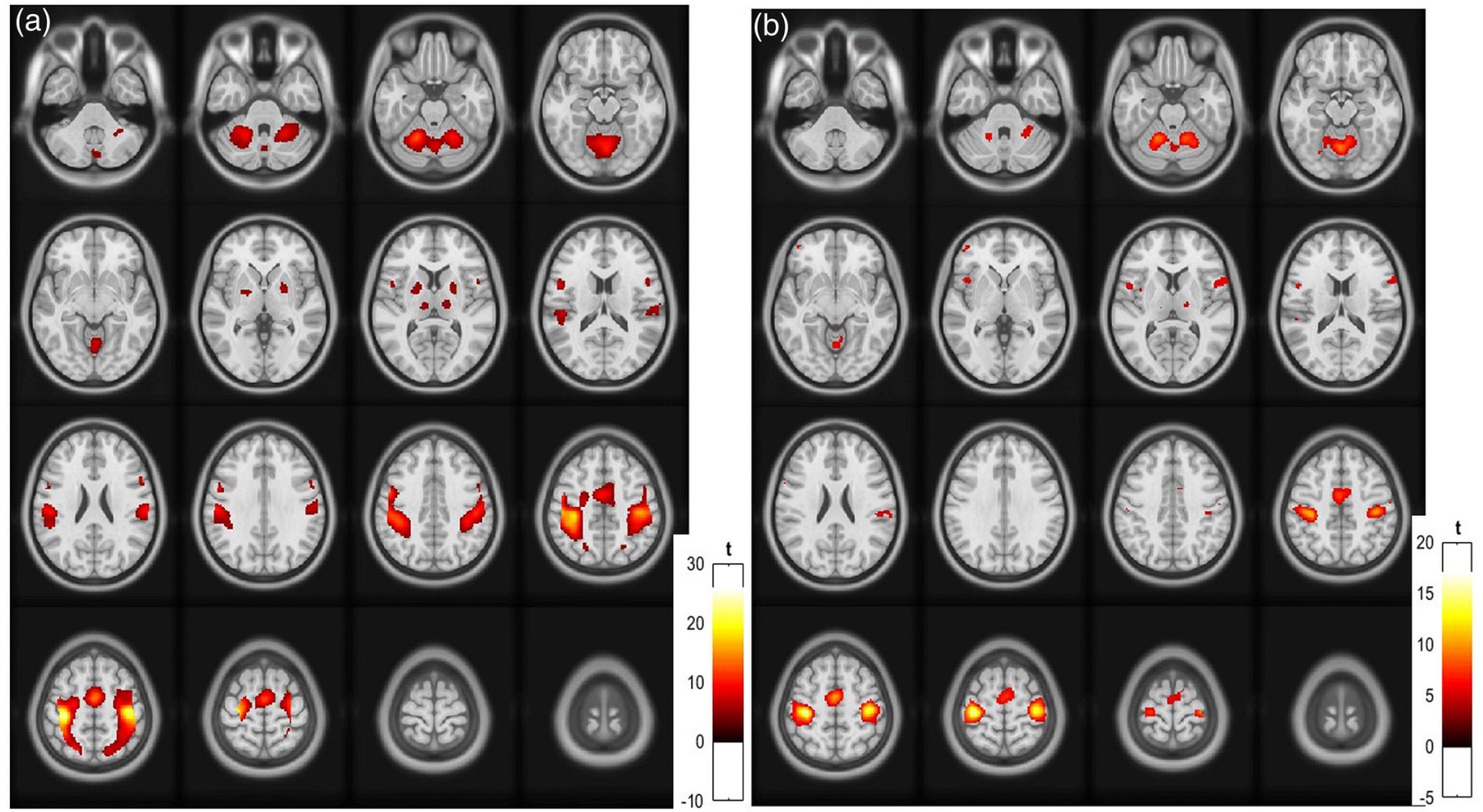

The contrasts in cerebral activations comparing melody vs. rest (a) and comparing rhythm vs. rest (b) were demonstrated. Activations occurring were demonstrated on 16 equispaced slices across the volume. (a) Significant activations were observed across cortical areas and the cerebellum. Bilateral activations in the primary sensorimotor cortices, the SMA, the anterior lobe of the cerebellum, PPC left thalamus and left putamen were observed $(P<0.001)$. (b) Activations were observed across cortical areas and the cerebellum. Bilateral activations in the primary sensorimotor cortices, the SMA, left thalamus and left putamen were observed. There were activations in the anterior lobe of the cerebellum, across superior vermis and paravermian areas, partially spreading to the cortices $(P<0.001)$. See color bar for the strength of activations. PPC, posterior parietal cortices; SMA, supplementary motor area.

Table $1 \mathrm{MNI}$ coordinates of local maxima for cerebral areas showing task-related activation in melody playing and rhythm groups. For melody-playing, fronto-parietal area spanned across precentral, postcentral regions, and supplementary motor area. Cerebellum anterior lobe includes vermis area for both tasks

\begin{tabular}{|c|c|c|c|c|c|c|c|c|c|}
\hline \multicolumn{5}{|c|}{ Melody-playing } & \multicolumn{5}{|c|}{ Rhythm } \\
\hline Region & $x, y, z$ & $T$ & $Z$ & $P$-cor & Region & $x, y, z$ & $t$ & $Z$ & $P$-cor \\
\hline Right fronto-parietal & $38,-26,62$ & 17.2 & 6.5 & 0.000 & Cerebellum anterior lob & $2,-62,-10$ & 13.8 & 6.0 & 0.000 \\
\hline Left fronto-parietal & $-34,-26,60$ & 13.5 & 6.0 & 0.000 & Right cerebellum & $12,-54,-20$ & 10.2 & 5.4 & 0.004 \\
\hline Cerebellum anterior lobe & $4,-60,-18$ & 11.8 & 5.7 & 0.001 & Left cerebellum & $-8,-58,-20$ & 8.6 & 5.0 & 0.026 \\
\hline Left cerebellum & $-16,-54,-24$ & 10.8 & 5.5 & 0.003 & Right SMA & $4,-10,54$ & 7.3 & 7.3 & 0.099 \\
\hline Right cerebellum & $14,-54,-24$ & 8.9 & 5.1 & 0.022 & Left SMA & $-5,-4,44$ & 7.3 & 4.6 & 0.108 \\
\hline Left putamen & $-22,4,8$ & 8.2 & 4.9 & 0.047 & Left fronto-parietal & $-36,-32,54$ & 6.8 & 4.5 & 0.177 \\
\hline Left thalamus & $-12,-18,10$ & 5.9 & 4.1 & 0.481 & Right fronto-parietal & $36,-28,56$ & 7.2 & 4.6 & 0.121 \\
\hline Left brainstem & $-6,-36,-44$ & 5.5 & 3.9 & 0.682 & Left thalamus & $-16,-16,8$ & 7.1 & 4.6 & 0.127 \\
\hline Left cerebellum posterior & $-12,-54,--40$ & 5.4 & 3.9 & 0.729 & Left temporal & $-42,2,8$ & 5.3 & 3.9 & 0.693 \\
\hline
\end{tabular}

MNI, Montreal Neurological Institute; $P$-cor, $P$-corrected; SMA, supplementary motor area.

To quantitatively assess the observed differences between melody-playing and rhythm tasks, activations were evaluated for melody-playing vs. rhythm and rhythm vs. melody-playing contrasts. In the melody-playing vs. rhythm contrast, significant activations were detected in PPC (Fig. 3a). No significant activations were detected in the rhythm vs. melody-playing contrast (Fig. 3b).
Laterality indices were calculated to assess hemispherical dominance during the melody-playing and rhythm tasks. Weak lateralization was observed in most of the brain regions with laterality indices in the range of laterality indices $\leq 0.2$, considered as bias. Only in the frontal lobe and basal ganglia laterality indices (respectively, laterality indices: $0.23 \pm 0.14$; laterality indices: $0.38 \pm 0.24$; $P<0.05)$ were slightly lateralized to left hemisphere in 


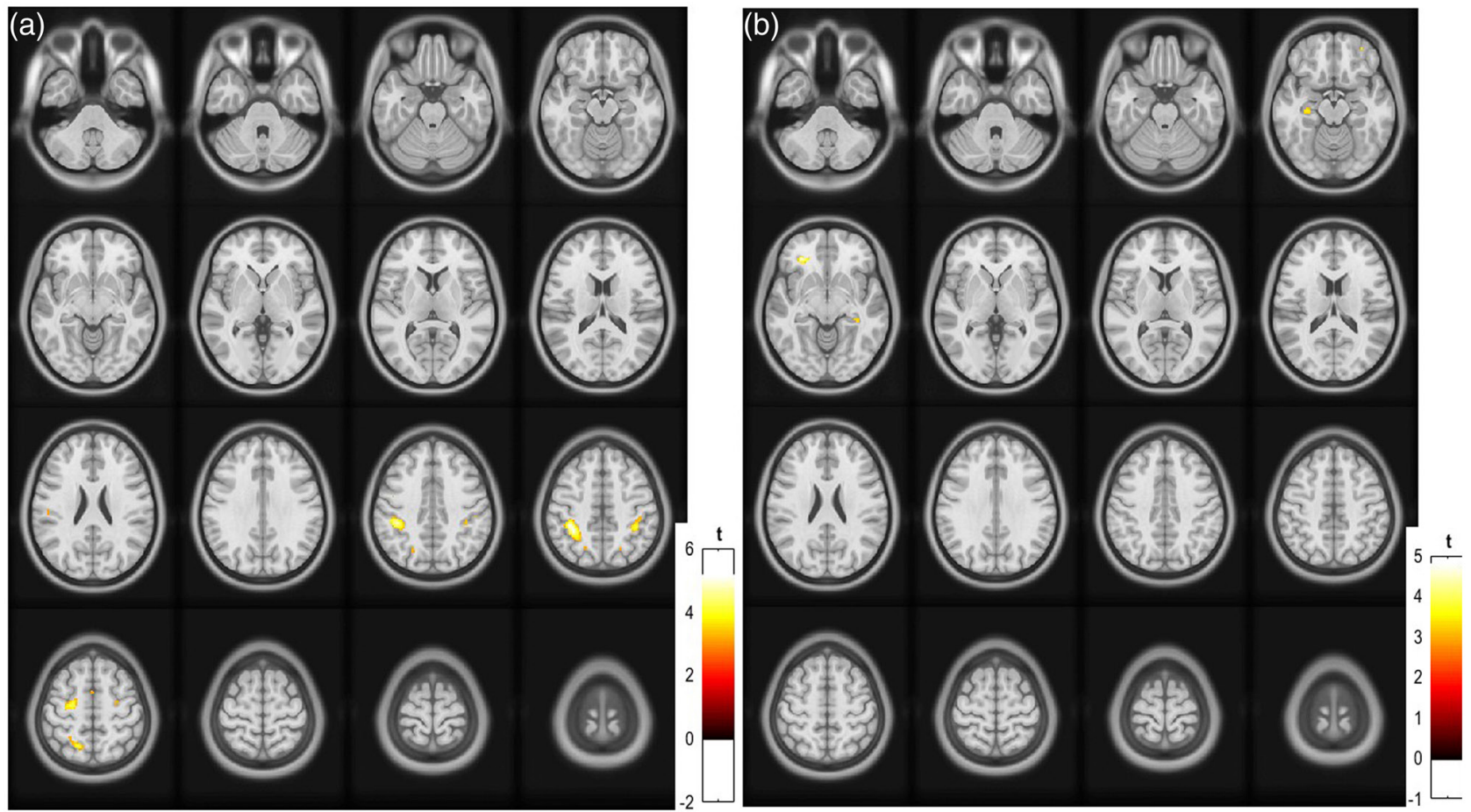

The contrasts in cerebral activations comparing melody-playing vs. rhythm (a) and comparing rhythm vs. melody-playing (b) were demonstrated. Activations occurring were demonstrated on 16 equispaced slices across the volume. (a) Significant activations were observed in PPC $(P<0.001)$. (b) No significant activations were observed $(P<0.001)$. See color bar for the strength of activations. PPC, posterior parietal cortices.

the melody-playing task, while parietal lobe, midline, and cerebellum were symmetric (respectively, laterality indices: $0.09 \pm 0.17$, laterality indices: $0.13 \pm 0.18$, laterality indices: $0.07 \pm 0.12 ; P>0.05)$. For rhythm, frontal lobe, parietal lobe, midline basal ganglia, and cerebellum have symmetric activity (respectively, $0.05 \pm 0.21,0.03 \pm 0.09$, $0.07 \pm 0.17,0.14 \pm 0.18,-0.17 \pm 0.20, P>0.05)$. To observe differences in hemispheric dominance between melody-playing and rhythm tasks laterality index differences were calculated. There were no significant differences in parietal, temporal, midline (e.g. SMA) cerebellar and basal ganglia laterality indices between melody-playing and rhythm tasks $(P>0.05)$. Only frontal laterality indices during rhythm was significantly lower (more symmetric) than the melody-playing task ( $z:-2.24 ; P=0.025)$.

DCM was performed to assess whether causal interactions existed between the left and right hemispheres during melody-playing and rhythm tasks. Action of melody-playing as a direct effect on both hemispheres was verified. The strength of hemodynamic responses with direct effects to left hemisphere (L1) and to right hemisphere (R1) was found to be 0.144 and $0.821 \mathrm{~Hz}$, respectively. The probability of a direct effect on L1 and R1 being present were both found to be 1 . Melody-playing was observed to be acting as a modulatory input from left to right hemisphere. No modulatory effect was observed in the right-to-left direction. The strength of hemodynamic responses with modulatory effects to left hemisphere (L1) and to right hemisphere (R1) were -0.079 and $2.586 \mathrm{~Hz}$, respectively. These results suggested that a strong modulatory effect was present from the left hemisphere to the right hemisphere. The probability of a modulatory effect from left-to-right was found to be 1 whereas the probability of a right-to-left modulatory effect remained below threshold at a probability of 0.736 . No causal interactions were observed during the rhythm task (see Fig. 1, Supplemental Digital Content 1, http:// links.lww.com/WNR/A582).

\section{Discussion}

Given that bimanual MSMs are relatively easy to control movements and the neural organization of pianists is more efficient as opposed to nonpianists (see for a broader discussion Text 4, Supplemental Digital Content 1, http://links.lwwe.com/WNR/A582), investigating different musical tasks such as melody-playing and rhythm could reveal findings regarding the neural underpinnings of these tasks which could have potential use in various neurophysiology applications. Driven by this motivation, this study aims to draw attention to the fact that different in-phase movements may have different bimanual interaction mechanisms, which may be of importance 
for neurophysiology literature and motor rehabilitation approaches. Here, we examined two different MSM tasks, comparing their anatomo-physiological features including hemispheric activations and dominance. In our study; primary sensorimotor cortices, SMA, anterior lobe of the cerebellum, putamen and thalamus were activated during both melody-playing and rhythm tasks. In particular, SMA was bilaterally activated during the sequential movement of either hand with no difference in the activation between the right and left hemispheres [23]. This finding is consistent with a previous study by Toyokura $e t$ al. [24], which suggests a larger role for the SMA proper in executive processing during bimanual, mirror performance of a complex movement. Meanwhile, left putaminal and thalamic activations in both groups were left lateralized. Previous studies suggest that putamen plays a role in movement initiation, motor programming, general attention or intention [25]. This role can be attributed to the serial connectivity of the basal ganglionic structure and the funneling of efferent projections to the ventrolateral thalamus and to the motor cortex [26].

In contrast, bilateral posterior parietal cortical activations were only detected during melody-playing. PPC activation is related to the performance of sequences [27]. Sequential finger movements as in melody-playing, where the sequence has to be performed from memory, are more complex than simple repetitive movements as in the rhythm task. Thus, the PPC activation observed here could be attributed to task complexity. This explanation is also in line with previous reports that underline the importance of increasing task complexity [28,29]. Another difference that the melody-playing task shows relate to hemispheric dominance. Here DCM analyses in melody-playing reveal that this task acts as a modulatory input on the left-to-right connection. Both DCM analysis and laterality indices have pointed out that the melody-playing task may be controlled by the dominant hemisphere. These results are consistent with a recent study that suggests that coordinated bilateral hand movements may be controlled from the dominant left hemisphere [30].

In the literature, bilateral cerebellar activation is not only attributed to sensory (e.g. auditory) or cognitive processing, but also to motor processing during music performance [31]. Motor topography is considered to be present in the anterior lobe of the contralateral cerebellum, closer to the vermis, and to partly extend into the hemispheres [32]. Specifically, it has been reported that the cerebellum, projecting to the counter-dorsal prefrontal cortex, modulates the high cognitive function during the executive process of contralateral cerebrocerebellar loop movement and modulates the acuity timing of previously learned behavior [33]. In alignment with these previous reports, here, simple repetitive movement (rhythm) and sequential complex movement (melody-playing) evoked activations in the vermian and paravermian areas of the anterior lobe of the cerebellum, and to a lesser degree in cerebral hemispheres.

Electroencephalography and transcranial magnetic stimulation studies report that as opposed to anti-phase, in-phase bimanual training increases M1 excitability [2]. The underlying neurophysiological mechanisms remain unclear [2,34-37] ('Text 5, Supplemental Digital Content 1, http://links.lww.com/WNR/A582) [38-44]. Corpus callosum is frequently used during movements of attention and bimanual learning, and callosal transmission affects the degree of bilateral synchrony $[23,45]$. In our study, because of large cortical activations in melody-playing, intercortical interaction via corpus callosum seems to be more apparent in melody-playing than rhythm. However, interaction via corpus callosum might be mostly based on inhibitory characteristics imposed by in-phase movements. When it is freed from the corpus callosum-related intercortical inhibition, especially in-phase bimanual movement may be made easier as in callosotomy and patients with split-brain [28]. On the other hand, patients with split-brain show difficulty in performing anti-phase bimanual movements in contrast to in-phase movement [42]. We argue that the melody-playing with MSM may contribute to bilateral cortical activation by using a different interaction way from anti-phase movement. Future studies are required for the verification of this argument (see Text 5, Supplemental Digital Content 1, http://links. lww.com/WNR/A582).

Here, we examined anatomo-physiological features of two MSM tasks in healthy subjects. An implicit motivation was that the proposed tasks could only be considered as potential candidates for rehabilitation if they produce significant activations in regions commonly associated with the respective motor deficits. Our findings indicate that the melody-playing task might be more relevant for deficits such as cortical infarcts associated with the primary motor cortex. Furthermore, the homologous information provided in bimanual melody-playing from the opposite hemisphere, and the subcortical control in rhythm might be beneficial in motor rehabilitation. Future studies are needed to evaluate the utility of MSM tasks in patient populations.

\section{Conclusion}

In this study, we examined the anatomo-physiological features of two musical tasks based on in-phase motor behavior: namely melody-playing and rhythm. In both melody-playing and rhythm tasks, bilateral activations were observed in sensorimotor and SMAs, cerebellum, putamen, and thalamus. Yet, symmetric activations of posterior parietal areas were only observed in the melody-playing task. While most activations were bilateral, thalamo-cortical circuit activations were left dominant. Activation in anterior lobe of the cerebellum, especially 
the superior vermis and paravermis, suggests that these areas serve a critical role in subcortical control of bimanual in-phase movements. In the melody-playing task, which likely leads to a different interhemispheric interaction via MSM, bilateral symmetrical activations were detected and classical right-hand information could be transferred to the left one in the first trial. Laterality index and DCM results indicate the presence of causal influences from the left to the right cerebral cortex.

\section{Acknowledgments \\ Conflicts of interest}

There are no conflicts of interest.

\section{References}

1 Cincotta M, Ziemann U. Neurophysiology of unimanual motor control and mirror movements. Clin Neurophysio/ 2008; 119:744-762.

2 Neva JL, Legon W, Staines WR. Primary motor cortex excitability is modulated with bimanual training. Neurosci Lett 2012; 514:147-151.

3 Koeneke S, Lutz K, Wüstenberg T, Jäncke L. Bimanual versus unimanual coordination: what makes the difference? Neuroimage 2004; 22:1336-1350.

4 Debaere F, Wenderoth N, Sunaert S, Van Hecke P, Swinnen SP. Changes in brain activation during the acquisition of a new bimanual coordination task. Neuropsychologia 2004; 42:855-867.

5 Serrien DJ. Coordination constraints during bimanual versus unimanual performance conditions. Neuropsychologia 2008; 46:419-425.

6 Maki Y, Wong KF, Sugiura M, Ozaki T, Sadato N. Asymmetric control mechanisms of bimanual coordination: an application of directed connectivity analysis to kinematic and functional MRI data. Neuroimage 2008; 42:1295-1304.

7 Rueda-Delgado LM, Solesio-Jofre E, Serrien DJ, Mantini D, Daffertshofer A, Swinnen SP. Understanding bimanual coordination across small time scales from an electrophysiological perspective. Neurosci Biobehav Rev 2014; 47:614-635.

8 Jäncke L, Shah NJ, Peters M. Cortical activations in primary and secondary motor areas for complex bimanual movements in professional pianists. Brain Res Cogn Brain Res 2000; 10:177-183.

9 Krings T, Töpper R, Foltys H, Erberich S, Sparing R, Willmes K, Thron A. Cortical activation patterns during complex motor tasks in piano players and control subjects. A functional magnetic resonance imaging study. Neurosci Lett 2000; 278:189-193.

10 Liuzzi G, Hörniss V, Zimerman M, Gerloff C, Hummel FC. Coordination of uncoupled bimanual movements by strictly timed interhemispheric connectivity. J Neurosci 2011; 31:9111-9117.

11 Aramaki Y, Honda M, Okada T, Sadato N. Neural correlates of the spontaneous phase transition during bimanual coordination. Cereb Cortex 2006; 16:1338-1348.

12 Laeng B, Park A. Handedness effects on playing a reversed or normal keyboard. Laterality 1999; 4:363-377.

13 Jäncke $L$. The case of a left-handed pianist playing a reversed keyboard: a challenge for the neuroscience of music. Neuroreport 2002; 13:1579-1583.

14 Jäncke L, Baumann S, Koeneke S, Meyer M, Laeng B, Peters M, Lutz K. Neural control of playing a reversed piano: empirical evidence for an unusual cortical organization of musical functions. Neuroreport 2006; 17:447-451.

15 Cattaert D, Semjen A, Summers JJ. Simulating a neural cross-talk model for between-hand interference during bimanual circle drawing. Biol Cybern 1999; 81:343-358.

16 Landau SM, D'esposito M. Sequence learning in pianists and nonpianists: an fMRI study of motor expertise. Cogn Affect Behav Neurosci 2006; 6:246-259.

17 Kelso JA. Phase transition and critical behavior in human bimanual coordination. Am J Physiol. 1984; 246:1000-1004.

18 Oldfield RC. The assessment and analysis of handedness: the Edinburgh inventory. Neuropsychologia 1971; 9:97-113.

19 Seghier ML. Laterality index in functional MRI: methodological issues. Magn Reson Imaging 2008; 26:594-601.
20 Wilke M, Lidzba K. LI-tool: a new toolbox to assess lateralization in functional MR-data. J Neurosci Methods 2007; 163:128-136.

21 Tzourio-Mazoyer N, Landeau B, Papathanassiou D, Crivello F, Etard O, Delcroix N, et al. Automated anatomical labeling of activations in SPM using a macroscopic anatomical parcellation of the MNI MRI single-subject brain. Neuroimage 2002; 15:273-289.

22 Friston KJ, Harrison L, Penny W. Dynamic causal modelling. Neuroimage 2003; 19:1273-1302.

23 Andres FG, Mima T, Schulman AE, Dichgans J, Hallett M, Gerloff C. Functional coupling of human cortical sensorimotor areas during bimanual skill acquisition. Brain 1999; 122 (Pt 5):855-870.

24 Toyokura M, Muro I, Komiya T, Obara M. Activation of pre-supplementary motor area (SMA) and SMA proper during unimanual and bimanual complex sequences: an analysis using functional magnetic resonance imaging. $J$ Neuroimaging 2002; 12:172-178.

25 Romo R, Scarnati E, Schultz W. Role of primate basal ganglia and frontal cortex in the internal generation of movements. II. Movement-related activity in the anterior striatum. Exp Brain Res 1992; 91:385-395.

26 Ropper $\mathrm{AH}$, Brown $\mathrm{RH}$. Abnormalities of movement and posture due to disease of the basal ganglia. In: Ropper AH, Brown RH, editors. Adams and Victor's Principles of Neurology. 8th ed. New York: McGraw-Hill Companies; 2005. p. 57.

27 Catalan MJ, Honda M, Weeks RA, Cohen LG, Hallett M. The functional neuroanatomy of simple and complex sequential finger movements: a PET study. Brain 1998; 121 (Pt 2):253-264.

28 Morris DM, Taub E, Mark VW. Constraint-induced movement therapy: characterizing the intervention protocol. Eura Medicophys 2006; 42:257-268.

29 Gerloff C, Andres FG. Bimanual coordination and interhemispheric interaction. Acta Psychol (Amst) 2002; 110:161-186.

30 Serrien DJ, Cassidy MJ, Brown P. The importance of the dominant hemisphere in the organization of bimanual movements. Hum Brain Mapp 2003; 18:296-305.

31 Jerde TA, Childs SK, Handy ST, Nagode JC, Pardo JV. Dissociable systems of working memory for rhythm and melody. Neuroimage 2011; 57:1572-1579.

32 Buckner RL, Krienen FM, Castellanos A, Diaz JC, Yeo BT. The organization of the human cerebellum estimated by intrinsic functional connectivity. $J$ Neurophysiol 2011; 106:2322-2345.

33 Obayashi S. Possible mechanism for transfer of motor skill learning: implication of the cerebellum. Cerebellum 2004; 3:204-211.

34 Carson RG. Neural pathways mediating bilateral interactions between the upper limbs. Brain Res Brain Res Rev 2005; 49:641-662.

35 Kwakkel G, Kollen BJ, Wagenaar RC. Long term effects of intensity of upper and lower limb training after stroke: a randomised trial. J Neurol Neurosurg Psychiatry 2002; 72:473-479.

36 Matsuda T, Watanabe S, Kuruma H, Murakamı Y, Watanabe R, Senou A. A comparison of three bimanual coordinations: an fMRI study. J Phys Ther Sci 2009; 21:85-92.

37 Sleimen-Malkoun R, Temprado JJ, Thefenne L, Berton E. Bimanual training in stroke: how do coupling and symmetry-breaking matter? BMC Neurol $2011 ; 11: 11$.

38 Mudie $\mathrm{MH}$, Matyas TA. Can simultaneous bilateral movement the undamaged hemisphere in reconstruction of neural networks damaged by stroke? Disabil Rehabil 2000; 22:23-37.

39 Neva JL, Vesia M, Singh AM, Staines WR. Modulation of left primary motor cortex excitability after bimanual training and intermittent theta burst stimulation to left dorsal premotor cortex. Behav Brain Res 2014; 261:289-296.

40 Diedrichsen J, Gush S. Reversal of bimanual feedback responses with changes in task goal. J Neurophysiol 2009; 101:283-288.

41 Pollok B, Butz M, Gross J, Schnitzler A. Intercerebellar coupling contributes to bimanual coordination. J Cogn Neurosci 2007; 19:704-719.

42 Ivry RB, Hazeltine E. Subcortical locus of temporal coupling in the bimanual movements of a callosotomy patients. Hum Mov Sci 1998; 18:345-375.

43 Meyer BU, Röricht S, Gräfin von Einsiedel H, Kruggel F, Weindl A. Inhibitory and excitatory interhemispheric transfers between motor cortical areas in normal humans and patients with abnormalities of the corpus callosum. Brain 1995; 118 (Pt 2):429-440.

44 Chan JL, Ross ED. Left-handed mirror writing following right anterior cerebral artery infarction: evidence for nonmirror transformation of motor programs by right supplementary motor area. Neurology 1988; 38 : 59-63.

45 Eliassen JC, Baynes K, Gazzaniga MS. Anterior and posterior callosal contributions to simultaneous bimanual movements of the hands and fingers. Brain 2000; 123 (Pt 12):2501-2511. 